\title{
Anti-K-RAS G12V mTCR-transduced Autologous Peripheral Blood Lymphocytes
}

National Cancer Institute

\section{Source}

National Cancer Institute. Anti-K-RAS G12V mT CR-transduced Autologous Peripheral

Blood Lymphocytes. NCI Thesaurus. Code C142888.

Autologous peripheral blood lymphocytes (PBLs) transduced with an HLA class I

histocompatibility antigen A*11:01 (HLA-A1101)-restricted murine T-cell receptor (mTCR)

that recognizes the glycine to valine point mutation at position 12 (G12V) variant of K-

RAS, with potential antineo plastic activity. HLA-A1101 positive PBLs are harvested from a K-RAS G12V-expressing cancer patient and transfected with a retroviral vector that encodes anti-K-RAS G12V mTCR. The transduced PBLs are then expanded in culture. When reintroduced to the patient, these anti-K-RAS G12V mTCR-expressing PBLs target and bind to K-RAS G12V-overexpressing tumor cells, which results in both cytokine secretion and tumor cell lysis. K-RAS, a member of the RAS family of oncogenes, serves an important role in cell signaling, division and differentiation. Mutation of K-RAS may induce constitutive signal transduction leading to tumor cell growth, proliferation, invasion, and metastasis. 